\title{
Observer Linearization for Nonlinear Systems Using Piecewise Multi-Linear Model
}

\author{
Tadanari Taniguchi and Michio Sugeno
}

\begin{abstract}
This paper proposes an observer design for piecewise nonlinear systems via observer linearization. The model is a piecewise multi-linear (PML) system, a nonlinear approximation, and fully parametric. Feedback linearization is applied to stabilize PML control system. However, since exact observer linearized conditions are more conservative than exact feedback linearized ones, the exact observer linearization can be applied to only a few nonlinear systems. This paper shows the PML model based linearized observer can be applied to a wider system than the conventional one. We apply the proposed method to TORA (Translational Oscillator with Rotating Actuator) system which is one of the benchmark problem for nonlinear control. Example is shown to confirm the feasibility of our proposals by computer simulation.
\end{abstract}

Keywords -observer linearization, piecewise multi-linear model, nonlinear control, feedback linearization

\section{Introduction}

Piecewise linear (PL) systems which are fully parametric have been intensively studied in connection with nonlinear systems [1], [2], [3], [4]. We are interested in the parametric piecewise approximation of nonlinear control systems based on the original idea of PL approximation. The PL approximation has general approximation capability for nonlinear functions with a given precision.

Sugeno suggested to use the piecewise multi-linear (PML) approximation [5]. PML approximation also has general approximation capability for nonlinear functions with a given precision. We note that a multi-linear function as a basis of PML approximation is, as a nonlinear function, the second simplest one after a linear function. The PML model has the following features. 1) The PML model is derived from fuzzy if-then rules with singleton consequents. 2) It is built on piecewise hyper-cubes partitioned in the state space. 3) It has general approximation capability for nonlinear systems. 4) It is a piecewise nonlinear model, the second simplest after a PL model. 5) It is continuous and fully parametric. The authors of this paper have been researching the PML systems [6-7], [9-12], [15-18].

This paper proposes an observer design for piecewise nonlinear systems via observer linearization. Feedback linearization can be applied to stabilize PML control system. However, since the observer linearized conditions are more conservative than feedback linearized ones the observer linearization can be applied to only a few nonlinear systems.
In comparison with the other observer designs [11], [12], this paper deals with the necessary and sufficient conditions for observer linearization. We show the PML model based linearized observer can be applied to a wider system than the conventional one. We apply the proposed method to TORA system which is one of the benchmark problem for nonlinear control.

This paper is organized as follows. Section II introduces the canonical form of PML models. Sections III and IV briefly present feedback linearization and observer linearization. Sections V and VI represent TORA system and the PML model. Sections VII and VIII propose the controllers and the observers of TORA system. Section IX shows an example demonstrating the feasibility of the proposed methods. Finally, section X summarizes conclusions.

\section{Canonical Forms of Piecewise Multi-Linear Models}

We introduce PML models suggested in [5]. We deal with the $n$-dimensional case of a nonlinear control system. Define vector $d\left(\sigma_{1}, \ldots, \sigma_{n}\right)$ and rectangle $R_{\sigma_{1} \cdots \sigma_{n}}$ in $n$ dimensional space as $d\left(\sigma_{1}, \ldots, \sigma_{n}\right) \equiv\left(d_{1}\left(\sigma_{1}\right), \ldots, d_{n}\left(\sigma_{n}\right)\right)^{T}$,

$$
\begin{aligned}
R_{\sigma_{1} \ldots \sigma_{n}} \equiv & {\left[d_{1}\left(\sigma_{1}\right), d_{1}\left(\sigma_{1}+1\right)\right] \times\left[d_{2}\left(\sigma_{2}\right), d_{2}\left(\sigma_{2}+1\right)\right] } \\
& \cdots \times\left[d_{n}\left(\sigma_{n}\right), d_{n}\left(\sigma_{n}+1\right)\right],
\end{aligned}
$$

where $\sigma_{i}$ is integer: $-\infty<\sigma_{i}<\infty, d_{i}\left(\sigma_{i}\right)<d_{i}\left(\sigma_{i}+1\right)$ and $d(0) \equiv\left(d_{1}(0), d_{2}(0), \ldots, d_{n}(0)\right)^{T}$. Superscript ${ }^{T}$ denotes a transpose operation.

We consider an $n$-dimensional nonlinear control system.

$$
\left\{\begin{array}{l}
\dot{x}=f(x)+g(x) u(x) \\
y=h(x)
\end{array}\right.
$$

For $x \in R_{\sigma_{1} \ldots \sigma_{n}}$, the PML model (2) is constructed from the nonlinear system (1).

$$
\left\{\begin{array}{l}
\dot{x}=f_{p}(x)+g_{p}(x) u(x) \\
y=h_{p}(x),
\end{array}\right.
$$

where

Tadanari Taniguchi

IT Education Center, Tokai University,

Hiratsuka, Kanagawa, 2591292 JAPAN 


$$
\left\{\begin{aligned}
f_{p}(x) & =\sum_{i_{1}=\sigma_{1}}^{\sigma_{1}+1} \cdots \sum_{i_{n}=\sigma_{n}}^{\sigma_{n}+1} \omega_{1}^{i_{1}}\left(x_{1}\right) \cdots \omega_{n}^{i_{n}}\left(x_{n}\right) f\left(i_{1}, \ldots, i_{n}\right), \\
g_{p}(x) & =\sum_{i_{1}=\sigma_{1}}^{\sigma_{1}+1} \cdots \sum_{i_{n}=\sigma_{n}}^{\sigma_{n}+1} \omega_{1}^{i_{1}}\left(x_{1}\right) \cdots \omega_{n}^{i_{n}}\left(x_{n}\right) g\left(i_{1}, \ldots, i_{n}\right), \\
h_{p}(x) & =\sum_{i_{1}=\sigma_{1}}^{\sigma_{1}+1} \ldots \sum_{i_{n}=\sigma_{n}}^{\sigma_{n}+1} \omega_{1}^{i_{1}}\left(x_{1}\right) \cdots \omega_{n}^{i_{n}}\left(x_{n}\right) h\left(i_{1}, \ldots, i_{n}\right), \\
x & =\sum_{i_{1}=\sigma_{1}}^{\sigma_{1}+1} \cdots \sum_{i_{n}=\sigma_{n}}^{\sigma_{n}+1} \omega_{1}^{i_{1}}\left(x_{1}\right) \cdots \omega_{n}^{i_{n}}\left(x_{n}\right) d\left(i_{1}, \ldots, i_{n}\right),
\end{aligned}\right.
$$

and $f\left(i_{1}, \ldots i_{n}\right), g\left(i_{1}, \ldots i_{n}\right), h\left(i_{1}, \ldots i_{n}\right)$, and $d\left(i_{1}, \ldots i_{n}\right)$ are the vertices of the nonlinear system (1). The membership functions are

$$
\left\{\begin{array}{l}
\omega_{j}^{\sigma_{j}}\left(x_{j}\right)=\frac{d_{j}\left(\sigma_{j}+1\right)-x_{j}}{d_{j}\left(\sigma_{j}+1\right)-d_{j}\left(\sigma_{j}\right)}, \\
\omega_{j}^{\sigma_{j}+1}\left(x_{j}\right)=\frac{x_{j}-d_{j}\left(\sigma_{j}\right)}{d_{j}\left(\sigma_{j}+1\right)-d_{j}\left(\sigma_{j}\right)},
\end{array}\right.
$$

$j=1, \ldots, n, \omega_{j}^{i}\left(x_{j}\right) \in\left[\begin{array}{ll}0 & 1\end{array}\right]$, and $x=\left(x_{1}, \ldots, x_{n}\right)^{T}$. In the above, we assume $f(0,0)=0$ and $d(0,0)=0$ to guarantee $\dot{x}=0$ for $x=0$.

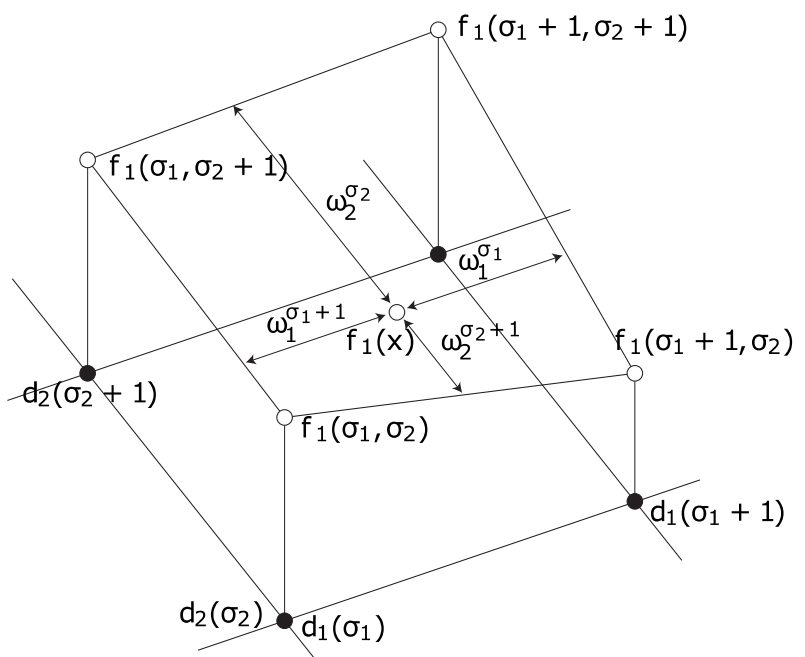

Figure 1: A piecewise region of $f_{1}(x)$ in $2 \mathrm{D}$ case

We explain the modeling procedure of PML system in two-dimensional case.

1) Assign vertices $d\left(i_{1}, i_{2}\right)$ for $x_{1}=d_{1}\left(\sigma_{1}\right), d_{1}\left(\sigma_{1}+1\right)$, $x_{2}=d_{2}\left(\sigma_{2}\right), d_{2}\left(\sigma_{2}+1\right)$ of state vector $x$, then partition state space into piecewise regions (see Fig. 1).

2) Compute vertices $f\left(i_{1}, i_{2}\right), g\left(i_{1}, i_{2}\right)$ and $h\left(i_{1}, i_{2}\right)$ in equation (3) by substituting values of $x_{1}=d_{1}\left(\sigma_{1}\right)$, $d_{1}\left(\sigma_{1}+1\right)$ and $x_{2}=d_{2}\left(\sigma_{2}\right), d_{2}\left(\sigma_{2}+1\right)$ into original nonlinear functions $f(x), g(x)$ and $h(x)$ in the system (1). Fig. 1 shows the expression of $f_{1}(x)$ and $x \in R_{\sigma_{1} \sigma_{2}}$.

The overall PML model is obtained automatically when all vertices are assigned. Note that $f(x), g(x)$ and $h(x)$ in the PML model coincide with those in the original system at vertices of all regions.

\section{Feedback Linearization}

This section deals with a feedback linearizing controller for PML model. Since the stabilizing conditions are represented by bilinear matrix inequalities (BMIs) [8], it requires a long computing time to obtain a stabilizing controller. To overcome the difficulty, we derived the stabilizing conditions [9], [10] based on feedback linearization approaches.

First we give a brief introduction to the feedback linearization [13] of nonlinear systems. We consider the nonlinear system (1), where $f(x), g(x)$ and $h(x)$ are assumed to be sufficiently smooth in a domain $D \subset R^{n}$. The mappings $f: D \rightarrow R^{n}$ and $D \rightarrow R^{n}$ are called vector fields on $D$. The time derivatives of the output $y$ are calculated until the input $u$ appears. Then the controller is obtained as

$$
u=\left(-L_{f}^{\rho} h(x)+v\right) / L_{g} L_{f}^{\rho-1} h(x) .
$$

The controller reduces the input-output map to $y^{(\rho)}=v$, which is a chain of $\rho$ integrators. In this case, the integer $\rho$ is called the relative degree of the system.

Definition 3.1: The nonlinear system is said to have relative degree $\rho, 1 \leq \rho \leq n$, in a region $D_{0} \subset D$ if

$$
\begin{aligned}
& L_{g} L_{f}^{i} h(x)=0, \quad i=0, \ldots \rho-2 \\
& L_{g} L_{f}^{i} h(x) \neq 0, \quad i=\rho-1
\end{aligned}
$$

for all $x \in D_{0}$. The feedback linearized system can be formulated as

$$
\left\{\begin{array}{l}
\dot{\xi}=A \xi+B v, \\
y=C \xi,
\end{array}\right.
$$

where

$$
\xi=\left(\begin{array}{c}
h \\
L_{f} h \\
\vdots \\
L_{f}^{\rho-2} h \\
L_{f}^{\rho-1} h
\end{array}\right), A=\left(\begin{array}{ccccc}
0 & 1 & 0 & \cdots & 0 \\
0 & 0 & 1 & \ddots & \vdots \\
\vdots & \vdots & \ddots & \ddots & 0 \\
0 & 0 & 0 & 0 & 1 \\
0 & 0 & 0 & 0 & 0
\end{array}\right), B=\left(\begin{array}{c}
0 \\
0 \\
\vdots \\
0 \\
1
\end{array}\right), C=\left(\begin{array}{c}
1 \\
0 \\
\vdots \\
0 \\
0
\end{array}\right)^{T}
$$

The stabilizing linear controller $v=-F \xi$ of the linearized system (4) can be obtained so that the transfer function $G=C(s I-A)^{-1} B$ is Hurwitz. Due to lack of space, this paper only deals with the case of $\rho=n$.

\section{Iv. Observer Linearization}

We consider the nonlinear system (1). If there exists a coordinate transformation $\zeta=\phi(x)$ such that the system (1) can be transformed into the following system:

$$
\begin{aligned}
& \dot{\zeta}=A_{0} \zeta+k(y)+r(y) u \\
& y=C_{0} \zeta
\end{aligned}
$$

with $\left(C_{0}, A_{0}\right)$ observable and $k, r: \mathfrak{R} \rightarrow \mathfrak{R}^{n}$ then it would be possible to build a full order state observer: 


$$
\begin{aligned}
& \dot{\hat{\zeta}}=A_{0} \hat{\zeta}+k(y)+H(\hat{y}-y) \\
& \hat{y}=C_{0} \hat{\zeta}
\end{aligned}
$$

The estimation error $e=\hat{\zeta}-\zeta$ satisfies the linear differential equation: $\dot{e}=\left(A_{0}+H C_{0}\right) e$. The estimation state is $\hat{x}=\phi^{-1}(\hat{\zeta})$. This problem is referred to as the observer linearization problem. The following theorem [13] gives a necessary and sufficient condition for the solution of the observer linearization problem.

Theorem 4.1: The observer linearization problem [13] is solvable if and only if there exists the neighborhood $V$ of an initial condition $x_{0}$ satisfies the following two conditions.

C1: $\operatorname{dim}\left(\operatorname{span}\left\{d h(x), \ldots d L_{f}^{n-1} h(x)\right\}\right)=n$, where $\forall x \in V$

C2: $\quad\left[a d_{f}^{i} \tau(x), a d_{f}^{j} \tau(x)\right]=0 \quad$,where $\quad 0 \leq i \leq n-1$, $0 \leq j \leq n-1, x \in V$.

The vector field $\tau(x)$ satisfies

$$
\left(d h(x)^{T}, d L_{f} h(x)^{T}, \ldots, d L_{f}^{n-1} h(x)^{T}\right)^{T} \tau(x)=(0, \ldots, 0,1)^{T}
$$

If the nonlinear system (1) is observer linearizable there exists a coordinate transformation $\zeta=\phi(x)$ satisfies the following condition.

$$
L_{(-1)^{j-1} a d_{f}^{j-1} \tau} \phi_{i}(x)= \begin{cases}0, & i \neq j \\ 1, & i=j\end{cases}
$$

The coordinate transformation $\zeta$ can be constructed as

$$
\zeta=\phi(x)=\left(\phi_{1}(x), \phi_{2}(x), \ldots, \phi_{n}(x)\right)^{T}
$$

\section{v. TORA System}

We consider TORA (Translational Oscillator with Rotating Actuator) system [14] which is one of the benchmark problem for nonlinear control. A coordinate transformation is applied to TORA system (Fig. 2) then the dynamics [14] is represented as

$$
\left\{\begin{array}{l}
\dot{x}=f(x)+g u=\left(\begin{array}{c}
x_{2} \\
-x_{1}+\varepsilon \sin x_{3} \\
x_{4} \\
0
\end{array}\right)+\left(\begin{array}{l}
0 \\
0 \\
0 \\
1
\end{array}\right) u \\
y=h(x)=x_{1}
\end{array}\right.
$$

where $x \in \mathfrak{R}^{4}, y \in \mathfrak{R}$, and the parameter $\varepsilon$ depends on the eccentricity, cart mass, and ball mass. In this paper, we apply the proposed methods to TORA system (6).

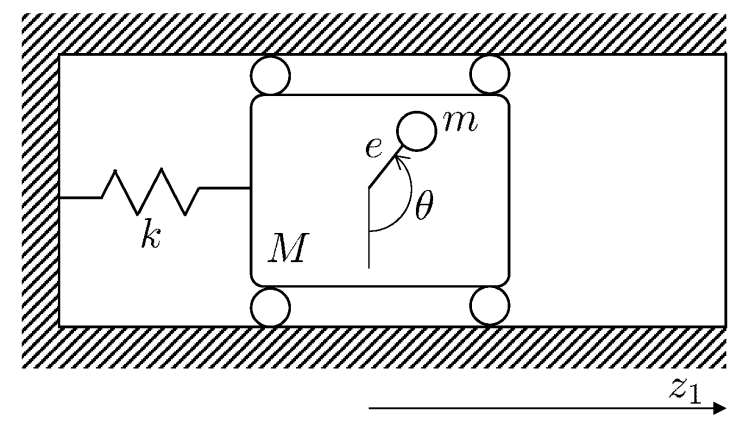

Figure 2: TORA system

\section{vI. PML Model of TORA System}

We construct the PML model of TORA system (6). The state variable $x$ is divided by $m_{1} \times m_{2} \times m_{3} \times m_{4}$ vertices,

$$
\begin{aligned}
& x_{1} \in\left\{d_{1}(1), \ldots, d_{1}\left(m_{1}\right)\right\}, x_{2} \in\left\{d_{2}(1), \ldots, d_{2}\left(m_{2}\right)\right\}, \\
& x_{3} \in\left\{d_{3}(1), \ldots, d_{3}\left(m_{3}\right)\right\}, x_{4} \in\left\{d_{4}(1), \ldots, d_{4}\left(m_{4}\right)\right\} .
\end{aligned}
$$

The PML model is expressed as (2), where $x \in R_{\sigma_{1} \ldots \sigma_{4}}$,

$$
f_{p}(x)=\sum_{i_{1}=\sigma_{1}}^{\sigma_{1}+1} \cdots \sum_{i_{4}=\sigma_{4}}^{\sigma_{4}+1} \omega_{1}^{i_{1}}\left(x_{1}\right) \cdots \omega_{4}^{i_{4}}\left(x_{4}\right)\left(\begin{array}{c}
d_{2}\left(i_{2}\right) \\
-d_{1}\left(i_{1}\right)+\varepsilon \sin d_{3}\left(i_{3}\right) \\
d_{4}\left(i_{4}\right) \\
0
\end{array}\right) \text {, }
$$

$g_{p}=\left(\begin{array}{llll}0 & 0 & 0 & 1\end{array}\right)^{T}$,

$\omega_{j}^{\sigma_{j}}\left(x_{j}\right)=\left(d_{j}\left(\sigma_{j}+1\right)-x_{j}\right) /\left(d_{j}\left(\sigma_{j}+1\right)-d_{j}\left(\sigma_{j}\right)\right)$,

$\omega_{j}^{\sigma_{j}+1}\left(x_{j}\right)=\left(x_{j}-d_{j}\left(\sigma_{j}\right)\right) /\left(d_{j}\left(\sigma_{j}+1\right)-d_{j}\left(\sigma_{j}\right)\right), j=1, \ldots, 4$.

The model is found to be fully parametric and the internal model dynamics is described by multi-linear interpolation of the vertices: $d_{1}\left(i_{1}\right), d_{2}\left(i_{2}\right), d_{3}\left(i_{3}\right)$ and $d_{4}\left(i_{4}\right)$ (see Fig. 1).

Note that there are some modeling errors because the PML model is a nonlinear approximation. In proposed method the vertices $d_{j}\left(i_{j}\right)$ of an arbitrary number can be set on arbitrary position of the state space. Therefore it is easily possible to adjust the approximated error.

\section{vII. Controller Designs for TORA System}

\section{A. Exact Feedback Linearization of Original Nonlinear Systems}

We design the controller of TORA system (6) via the exact feedback linearization [13]. The time derivatives of the output $y$ have to be calculated until the input $u$ appears. Then the controller is obtained as

$$
u=\frac{-x_{1}+\varepsilon \sin x_{3}+\varepsilon x_{4}^{2} \sin x_{3}}{\varepsilon \cos x_{3}}+\frac{1}{\varepsilon \cos x_{3}} v
$$

where $v$ is the linear controller for the following linearized system (4), where $\xi=\left(h, L_{f} h, L_{f}^{2} h, L_{f}^{3} h\right)^{T}$. The parameters $A$, $B$, and $C$ are the same as the system (4).

However, the controller (7) is only well defined at $-\pi / 2<x_{3}<\pi / 2$ because the denominator of the controller is $\varepsilon \cos x_{3}$. Hence the rotor $x_{3}$ of TORA system can only be rotated at $-\pi / 2<x_{3}<\pi / 2$.

\section{B. Exact Feedback Linearization of PML Systems}

We design the controller via the exact feedback linearization using the PML model (2) of TORA system. The time derivatives of the output $y$ also have to be 
calculated until the input $u$ appears. Then the controller is obtained as

$$
\begin{aligned}
u & =\left(-L_{f_{p}}^{4} h_{p}+v\right) / L_{g_{p}} L_{f_{p}}^{3} h_{p} u \\
= & \frac{\sum_{i_{1}=\sigma_{1}}^{\sigma_{1}+1} \sum_{i_{3}=\sigma_{3}}^{\sigma_{3}+1} \omega_{1}^{i_{1}}\left(x_{1}\right) \omega_{3}^{i_{3}}\left(x_{3}\right)\left(-d_{1}\left(i_{1}\right)+\varepsilon \sin d_{3}\left(i_{3}\right)\right)}{\varepsilon\left(\sin d_{3}\left(i_{3}+1\right)-\sin d_{3}\left(i_{3}\right)\right) /\left(d_{3}\left(i_{3}+1\right)-d_{3}\left(i_{3}\right)\right)}(8) \\
& -\frac{d_{3}\left(i_{3}+1\right)-d_{3}\left(i_{3}\right)}{\varepsilon\left(\sin d_{3}\left(i_{3}+1\right)-\sin d_{3}\left(i_{3}\right)\right)} v
\end{aligned}
$$

where $v=-F \xi$ is the linear controller of the linear system (4), where $\xi=\left(h, L_{f} h, L_{f}^{2} h, L_{f}^{3} h\right)^{T}$. The parameters $A, B$, and $C$ are the same as the system (4). If $\sin d_{3}\left(i_{3}+1\right) \neq \sin d_{3}\left(i_{3}\right)$, $i_{3}=1, \ldots, m_{3}$, there exists a PML controller (8) of TORA system (6) at $\forall x_{3}$ since $L_{g_{p}} L_{f_{p}}^{3} h_{p} \neq 0$. Thus we have to construct the PML model of TORA system such that $\sin d_{3}\left(i_{3}+1\right) \neq \sin d_{3}\left(i_{3}\right)$. Therefore the PML model based controller (8) can be applied to a wider region than the conventional feedback linearized controller (7).

\section{Observer Designs for TORA System}

\section{A. Exact Observer Linearization of Original Nonlinear System}

C1 of Theorem 4.1 is calculated for the original nonlinear system (6).

$$
\operatorname{det} D_{1}=\varepsilon^{2} \cos ^{2} x_{3}
$$

where $D_{1}=\left(d h(x)^{T} d L_{f} h(x)^{T}, \ldots, d L_{f}^{n-1} h(x)^{T}\right)^{T}$. The matrix $D_{1}$ is not linear independence at $x_{3}= \pm \pi / 2$. One of the condition $\mathrm{C} 2$ is also calculated for the original nonlinear model as follows:

$$
\left[a d_{f}^{0} \tau(x), a d_{f}^{3} \tau(x)\right]=\frac{2 \sin x_{3}}{\varepsilon^{2} \cos ^{3} x_{3}}
$$

The above equation is equal to 0 at $x_{3}=0$ and the equation cannot be defined at $x_{3}= \pm \pi / 2$. Therefore the nonlinear system (6) is not observer linearizable.

\section{в. Exact Observer Linearization of PML System}

C1 of Theorem 4.1 is calculated for the PML system (2) of TORA system (6).

$$
\operatorname{det}\left(d h(x)^{T} d L_{f} h(x)^{T}, \ldots, d L_{f}^{n-1} h(x)^{T}\right)^{T}=a \neq 0,
$$

where $a$ is a non-negative constant value. C2 of Theorem 4.1 is also calculated for the PML model (2) of TORA system (6).

$$
\left[a d_{f}^{i} \tau(x), a d_{f}^{j} \tau(x)\right]=0,
$$

where $0 \leq i \leq 3,0 \leq j \leq 3$ and $\tau(x)=\left(\begin{array}{llll}0 & 0 & 0 & 1 / a\end{array}\right)^{T}$. Therefore the PML system ( 3 ) is observer linearizable. From the conditions (5), the coordinate transformation vector $\zeta=\phi(x)=\left(\begin{array}{llll}a x_{4} & a x_{3} & x_{2} & x_{1}\end{array}\right)^{T}$ can be constructed. We can also design the observer gain $H$ such that the estimation error system $\dot{e}=\left(A_{0}+H C_{0}\right) e$ is stable.

\section{Simulation result}

We design the PML model based linearized controller (8) and the observer for TORA system (6) in a computer simulation. In this simulation, the state variables $x_{1}, x_{2}, x_{3}$, and $x_{4}$ of TORA system are divided by the following vertices.

$$
\begin{gathered}
x_{1} \in\{-2.5,0,2.5\}, x_{2} \in\{-2.5,0,2.5\} \\
x_{3} \in\{-\pi,-7 \pi / 8, \ldots, \pi\}, x_{4} \in\{-10,0,10\}
\end{gathered}
$$

The parameter $\varepsilon$ is 0.5 . The control system parameters are as follows: the feedback gain is

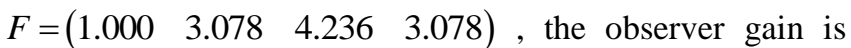
$H=\left(\begin{array}{llll}316.2 & 764.4 & 765.9 & 318.6\end{array}\right)^{T}$, and the observer system matrices are

$$
A_{0}=\left(\begin{array}{llll}
0 & 0 & 0 & 0 \\
1 & 0 & 0 & 0 \\
0 & 1 & 0 & 0 \\
0 & 0 & 1 & 0
\end{array}\right), C_{0}=\left(\begin{array}{l}
0 \\
0 \\
0 \\
1
\end{array}\right)^{T} .
$$

The initial conditions are $x(0)=\left(\begin{array}{llll}1 & 0 & 0 & 0\end{array}\right)^{T}$ and $\zeta(0)=\left(\begin{array}{llll}0 & 0 & 0 & 0\end{array}\right)^{T}$. Figs. 3 and 4 show the linearized observer states and the estimated states. Fig. 5 shows the state responses.
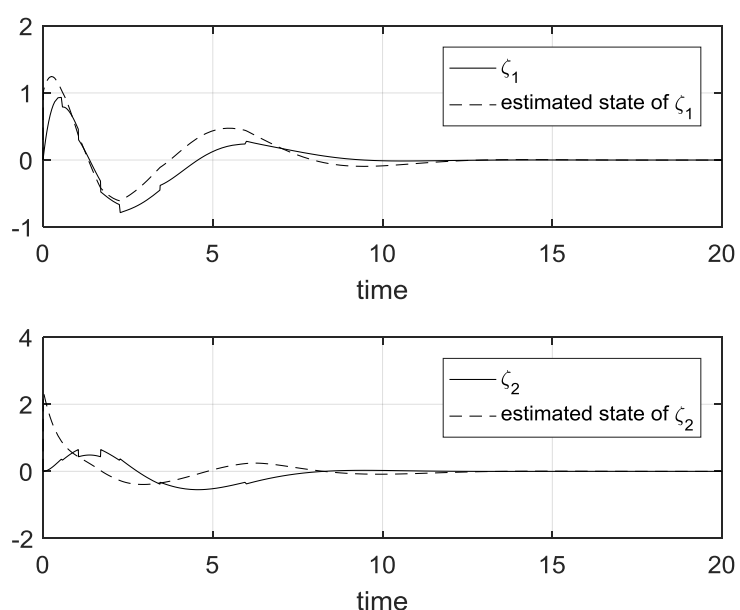

Figure 3: Linearized observer states $\left(\zeta_{1}, \zeta_{2}\right)$ and the estimations of TORA system 

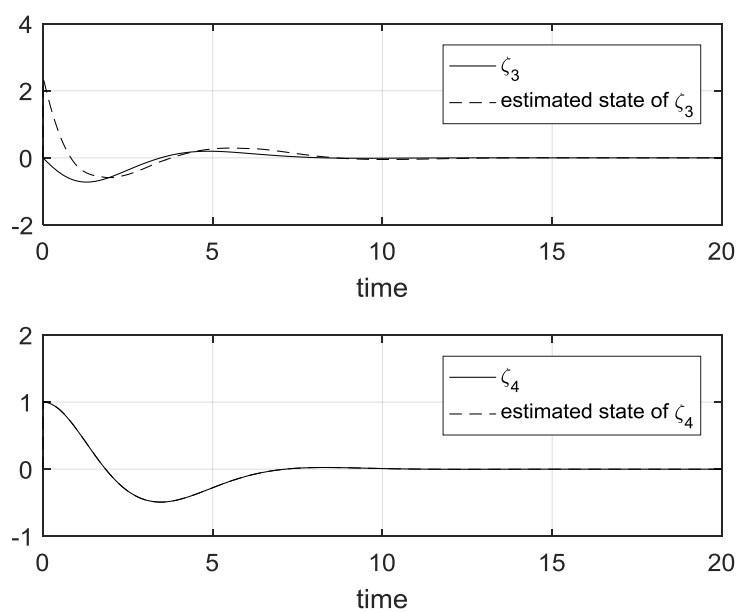

Figure 4: Linearized observer states $\left(\zeta_{3}, \zeta_{4}\right)$ and the estimations of TORA system
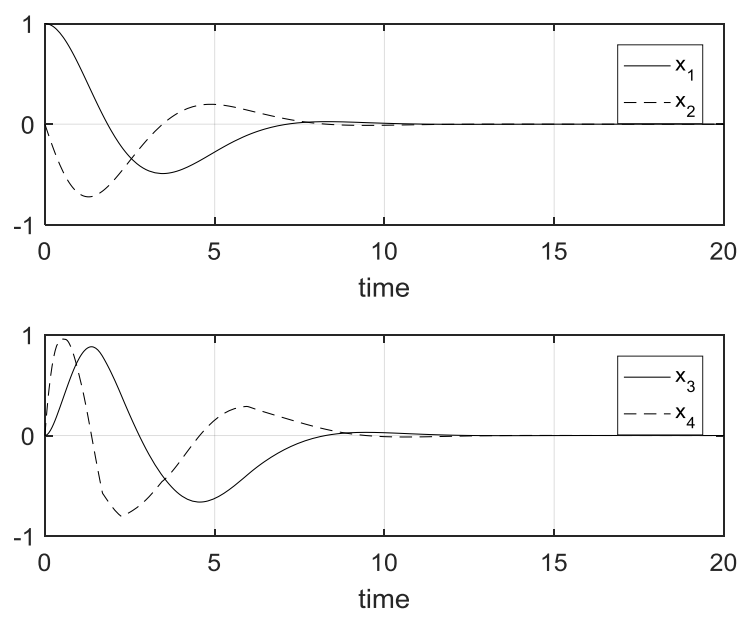

Figure 5: State responses of TORA system

\section{x. Conclusion}

This paper has proposed an observer design for piecewise nonlinear systems via observer linearization. The model is a PML system, a nonlinear approximation, and fully parametric. Feedback linearization can be applied to stabilize PML control systems. However, since exact observer linearized conditions are more conservative than exact feedback linearized ones, the exact observer linearization can be applied to only a few nonlinear systems. This paper has showed the PML model based linearized observer could be applied to a wider system than the conventional one. We have applied the proposed method to TORA system which was one of the benchmark problem for nonlinear control. Example has been shown to confirm the feasibility of our proposals by computer simulation.

In future work, we will apply the proposed methods to real systems and will design an observer-based feedback controller for nonlinear systems using PML models.

\section{References}

[1] E. D. Sontag, "Nonlinear regulation: the piecewise linear approach," IEEE Trans. Autom. Control, vol. 26, pp. 346-357, 1981.

[2] M. Johansson and A. Rantzer, "Computation of piecewise quadratic, lyapunov functions of hybrid systems," IEEE Trans. Autom. Control, vol. 43, no. 4, pp. 555-559, 1998.

[3] J. Imura and A. van der Schaft, "Characterization of well-posedness of piecewise-linear systems," IEEE Trans. Autom. Control, vol. 45, pp. 1600-1619, 2000.

[4] G. Feng, G. P. Lu, and S. S. Zhou, "An approach to hinfinity controller synthesis of piecewise linear systems," Communications in Information and Systems, vol. 2, no. 3, pp. 245-254, 2002.

[5] M. Sugeno, "On stability of fuzzy systems expressed by fuzzy rules with singleton consequents," IEEE Trans. Fuzzy Syst., vol. 7, no. 2, pp. 201-224, 1999.

[6] M. Sugeno and T. Taniguchi, "On improvement of stability conditions for continuous mamdani-like fuzzy systems," IEEE Tran. Systems, Man, and Cybernetics, Part B, vol. 34, no. 1, pp. 120-131, 2004.

[7] T. Taniguchi and M. Sugeno, "Stabilization of nonlinear systems based on piecewise lyapunov functions," in FUZZ-IEEE 2004, 2004, pp. $1607-1612$.

[8] K.-C. Goh, M. G. Safonov, and G. P. Papavassilopoulos, "A global optimization approach for the BMI problem," in Proc. the 33rd IEEE CDC, 1994, pp. 2009-2014.

[9] T. Taniguchi and M. Sugeno, "Piecewise bilinear system control based on full-state feedback linearization," in SCIS \& ISIS 2010, 2010, pp. 1591-1596.

[10] — "Stabilization of nonlinear systems with piecewise bilinear models derived from fuzzy if-then rules with singletons," in FUZZIEEE 2010, 2010, pp. 2926-2931.

[11] T. Taniguchi, L. Eciolaza, and M. Sugeno, "Full-order state observer design for nonlinear systems based on piecewise bilinear models," International Journal of Modeling and Optimization, vol. 4, no. 2, pp. 120-125, 2014.

[12] - "Designs of minimal-order state observer and servo controller for a robot arm using piecewise bilinear models," in Proceeding of The 2014 IAENG International Conference on Control and Automation. IAENG, 2014.

[13] S. Sastry, "Nonlinear Systems, Analysis, Stability, and Control," Springer, IEEE Trans. Autom. Control, vol. 30, no. 3, pp. 258-265, 1985.

[14] R. Sepulchre, M. Jankovic, and P. Kokotovic, Constructive Nonlinear Control. Springer, 1999.

[15] T. Taniguchi and M. Sugeno, "Trajectory Tracking Controller Design for a Tricycle Robot Using Piecewise Multi-Linear Models", in Proceeding of The 2017 IAENG International Conference on Control and Automation. IAENG, 2017.

[16] T. Taniguchi and M. Sugeno, "Piecewise Multi-Linear Model Based Control for TORA System via Feedback Linearization", in Proceeding of The 2018 IAENG International Conference on Control and Automation. IAENG, 2018.

[17] T. Taniguchi and M. Sugeno, "Piecewise Multi-Linear Model Based Lookup Table Controller for Nonlinear Systems with Input Constraints", in Proceeding of World Conference on Soft Computing 2018, 2018.

[18] T. Taniguchi and M. Sugeno, "Robust Lookup Table Controller Based on Piecewise Multi-Linear Model for Nonlinear Systems with Parametric Uncertainty", in Proceeding of 17th International Conference on Information Processing and Management of Uncertainty in Knowledge-Based Systems, 2018

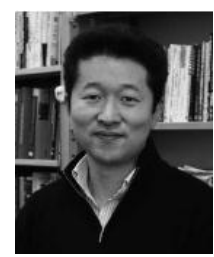

Tadanari Taniguchi received the B.S. and M.S. degrees in mechanical systems engineering from Kanazawa University, Kanazawa, Japan, in 1996 and 1998 respectively, and the $\mathrm{Ph} . \mathrm{D}$. degree in mechanical and control engineering from University of ElectroCommunications, Tokyo, Japan, in 2001. From 2001 to 2005, he was a Research Scientist of the Brain Science Institute, RIKEN, Saitama, Japan. Since 2005, he has been with IT Education Center, Tokai University, Kanagawa, Japan. From 2015 to 2016, he was a Visiting Researcher of Université de Valenciennes, France. He is currently an Associate Professor with Tokai 
University. His research interests include intelligent control, nonlinear control, feedback error learning and expert system.

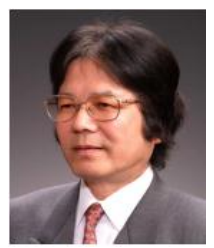

Michio Sugeno After graduating from the Department of Physics, The University of Tokyo, He worked at Mitsubishi Atomic Power Industry. T hen, he served the Tokyo Institute of Technology as Research Associate, Associate Professor and Professor from 1965 to 2000. After retiring from the Tokyo Institute of Technology, he worked as Laboratory Head at the Brain Science Institute, RIKEN from 2000 to 2005, as Distinguished Visiting Professor at Doshisha University from 2005 to 2010, and then, as Emeritus Researcher at the European Centre for Soft Computing, Spain from 2010 to 2015. He is currently Emeritus Professor at the Tokyo Institute of Technology, Japan. He was as President of the Japan Society for Fuzzy Theory and Systems from 1991 to 1993, and also President of the International Fuzzy Systems Association from 1997 to 1999. He is the first recipient of the IEEE Pioneer. Award in Fuzzy Systems with Zadeh in 2000. He also received the 2010 IEEE Frank Rosenblatt Award and recently Kampt de Ferit Award in 2012. 